\title{
Imaging Bacterial Cell Death Induced by Antimicrobial Peptides in Real Time Using High Speed AFM
}

\author{
Georg E. Fantner†, Roberto J. Barbero*, David S. Gray†, Angela M. Belcher†*
}

$\dagger$ Biomolecular Materials Group, Department of Materials Science and Engineering, Massachusetts Institute of Technology, Cambridge MA, 02139

*Biomolecular Materials Group, Department of Biological Engineering, Massachusetts Institute of Technology, Cambridge MA, 02139

Antimicrobial peptides (AMP) are a promising class of antimicrobial agents in the battle against bacteria that have built up resistance to conventional antibiotics. The mechanism by which these peptides kill bacteria is still poorly understood. The interaction of the peptides with the bacterial cell walls has been suggested to be responsible for the bactericidal effect [1,2,3]. Thus far, this interaction has not been visualized nor is there much data on the kinetics of this interaction. Traditional Atomic Force Microscopy (AFM) is a useful tool for observing the changes in the cell wall of living cells [4], but due to its slow image acquisition speed it cannot provide insight into the dynamics of the mechanism. We have used custom-built AFM components based on small cantilevers [5] to image for the first time the bactericidal action of antimicrobial peptides in real time. With this system, we investigated the activity of the chimeric AMP CM15 with nanometer spatial- and seconds temporal-resolution on live E.coli cells. We observed rapid changes in surface morphology of the cells after injection of the AMP (see figure 1), with a response time that differs between individual monoclonal bacteria in the same image (see figure 2). Using combined AFM and fluorescence microscopy, we correlated the change in cell morphology to cell wall permeability and cell death [6]. The results of this study show that high-speed atomic force microscopy can reveal distinctively different behavior of individual cells than compared to the behavior of bulk cells. In the case of the antimicrobial peptides, the presence of an incubation-phase gives new indications for the mechanism by which the antimicrobial peptide attacks the bacterium. We believe that this technique will enable a whole new method of characterizing and studying the effectiveness of synthetic antimicrobial peptides.

\section{References}

[1] B. Bechinger, Biochimica Et Biophysica Acta-Biomembranes 1462, 157 (Dec 15, 1999).

[2] G. Francius, O. Domenech, M. P. Mingeot-Leclercq, Y. F. Dufrene, Journal of Bacteriology 190, 7904 (December 15, 2008, 2008).

[3] R. E. W. Hancock, Current drug targets - Infectious disorders 2, 79 (2002).

[4] Y. F. Dufrene, D. J. Muller, Microbial Imaging 34, 163 (2005).

[5] G. E. Fantner et al., Ultramicroscopy 106, 881 (Jun-Jul, 2006).

[6] G. E. Fantner, R. J. Barbero, G. S. Gray, A. M. Belcher, Nature Nanotechnology In press, (2010). 

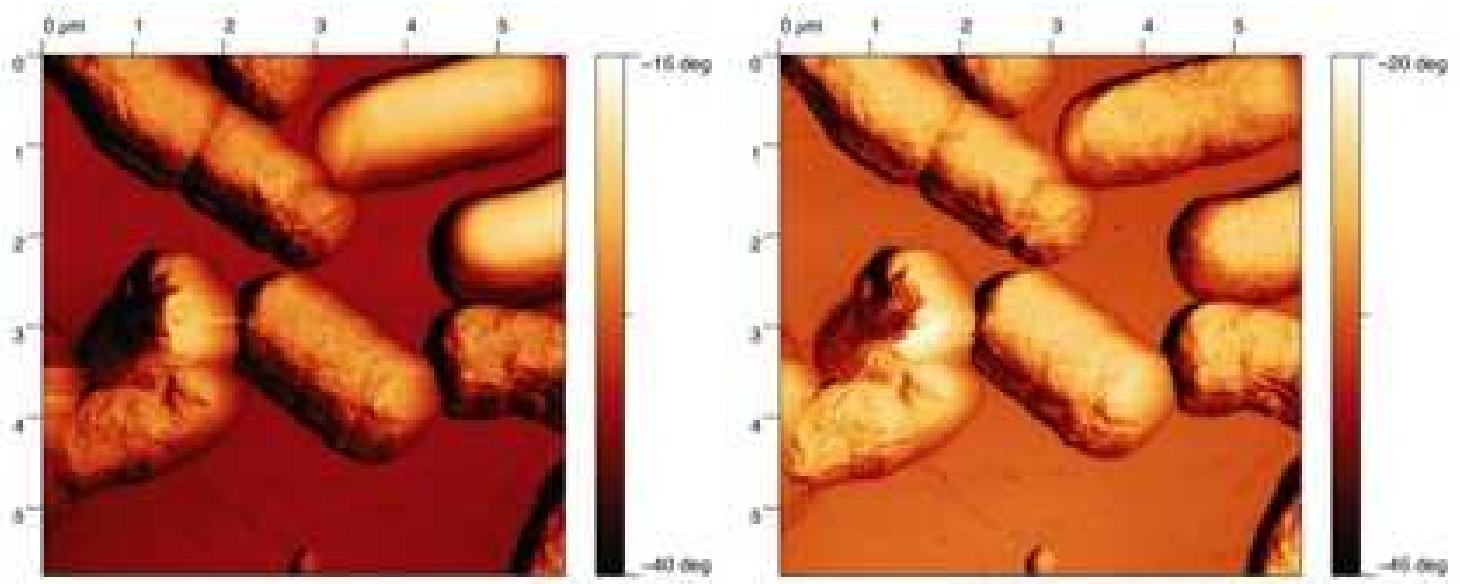

Figure 1: Bacterial response to antimicrobial peptides. Left: some bacteria have been attacked by the AMP (rough bacterial surfaces) but some still resist the antibiotic (smooth bacteria upper right corner). Right: 7 seconds later all bacteria are affected.

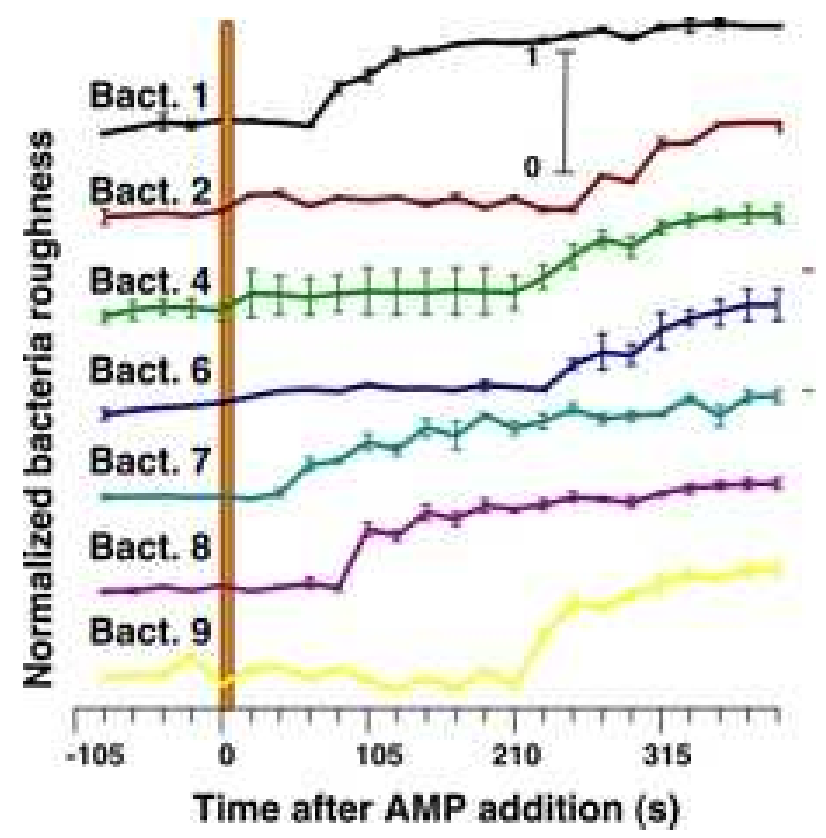

Figure 2: Variations onset time for bacterial cell death induced by antimicrobial peptides. The cells respond to the antimicrobial peptide on different time scales. The time it takes for the bacterium to be fully affected by the peptide consists of a time variant incubation phase (flat parts of the curves) and a time invariant active phase (increasing parts of the curve). 